\title{
Genetic factors in the presence, severity, and triggers of asthma
}

\author{
Edward P Sarafino, Jarrett Goldfedder
}

\begin{abstract}
The role of heredity in the presence of asthma, severity of the condition, and impact of 12 specific triggers of attacks was investigated. Health surveys containing questions about children's asthma characteristics were completed by 325 families with twin children across the United States. Data for 39 monozygotic twin pairs and 55 same sex dizygotic twin pairs who were between 2 and 20 years of age and had asthma present in at least one member of each pair were received and analysed. Results revealed higher concordance for the presence of asthma among monozygotic $(58.97 \% ; n=23)$ than dizygotic twins $(23 \cdot 64 \% ; n=13)$. Further analyses were restricted to data from the concordant monozygotic and dizygotic twin pairs. Asthma severity (the product of attack frequency and intensity ratings) was significantly correlated for monozygotic pairs but not for dizygotic pairs, and this difference in monozygotic and dizygotic severity correlations was significant. Also, monozygotic twins showed significantly higher correlations than dizygotic twins for the impacts of two asthma triggers: respiratory infection and physical activity. These results indicate a role of heredity in the presence of asthma and suggest that genetic factors may also affect the severity of children's asthma condition and the impact of respiratory infection and physical exertion as asthma triggers.

(Arch Dis Child 1995; 73: 112-116)
\end{abstract}

Keywords: asthma, genetics, severity, triggers.

Asthma is a chronic respiratory disorder characterised by recurrent episodes of impaired breathing when the airways become obstructed. ${ }^{1}$ This disease is highly prevalent around the world - in the United States, it afflicts about 10-12 million people and results in 500000 hospitalisations and 5000 deaths annually. ${ }^{2}$ Although people can develop asthma at any age, its childhood prevalence rates appear to be highest in the early grade school years, particularly among boys. ${ }^{3} 4$

Considerable evidence exists that heredity plays a part in the development of asthma. Twin studies have demonstrated higher rates of concordance among monozygotic than dizygotic twins for the presence of asthma..$^{5-8}$ Family history studies have found that prevalence rates of asthma are higher among relatives of asthmatic than non-asthmatic children and that the respiratory capacities of parents and children are correlated, which may reflect hereditary control..$^{10}$ Twin and family history studies have also reported evidence for genetic connections to serum IgE concentrations ${ }^{11-14}$ and to respiratory sensitivity under exercise challenge ${ }^{15-17}$ and acetylcholine challenge. ${ }^{18} 19$ In addition, recent genetic research on another disease, myotonic dystrophy, found that the size of the genetic fragment causing that condition becomes larger across generations and is correlated with the severity of the condition..$^{20}$ Taken together, these findings suggest the possibility that genetic factors may influence not only the presence of asthma, but the severity of the asthma condition, as well.

Asthma episodes tend to occur in conjunction with certain circumstances, or triggers, and the triggering effects of these circumstances vary from one asthmatic to the next as well as within the same individual across time. ${ }^{21-24}$ Common triggers of asthma attacks include airborne or ingested allergens, environmental temperature and humidity, night time hours, air pollution and cigarette smoke, strenuous exercise, viral respiratory infections, and emotional states or behaviours, such as anxiety or laughter. Given the established role of genetic factors in respiratory sensitivity to exercise and acetylcholine challenge, it seems likely that heredity influences the impact of specific triggers in precipitating asthma attacks.

The data reported here examine the role of genetic factors in the presence of asthma, severity of the asthma condition, and the specific triggers of asthma attacks. These data represent part of the findings from the Family Health Project in which a wide variety of biological and psychosocial factors were studied for several childhood illnesses, particularly asthma, using a relatively exploratory methodology. Parents of twins from across the United States were asked to complete a questionnaire that asked about their twin children's asthma presence, severity, and triggers. We hypothesised that monozygotic twins would be more similar than dizygotic twins for all three of these variables.

\section{Subjects and methods} SUBJECTS

Families $(n=325)$ with twin children were recruited through the National Organization of Mothers of Twins Clubs in the United States to participate in the Family Health Project. The clubs were asked to encourage all members to participate, especially those with family histories of asthma. To control for possible 
biases in the data, only same sex twins with at least one asthmatic member between the ages of 2 and 20 years were included in the current analyses. Although some children develop asthma in infancy, distinguishing between asthma and respiratory infections before 2 years of age is difficult, and most people who will develop asthma do not experience the onset until after 2 or 3 years of age. ${ }^{425}$ The final sample contained 23 male and 16 female monozygotic pairs and 27 male and 28 female dizygotic pairs, with overall mean (SD) ages of $7.9(5.0)$ years for monozygotic and $7 \cdot 2(3.6)$ years for dizygotic twins.

\section{MATERIALS AND PROCEDURE}

The researchers prepared a questionnaire to assess the health and health related practices of individuals within each family and received approval from the institutional review board to conduct the study. A parent (typically the mother) from each family completed the survey and returned it to the researchers either directly or through the club officers. If the family had more than one twin pair, the oldest pair was selected as subjects for this survey. If necessary, a respondent could select a twin pair from a multiple birth (for example, triplets) using an unbiased procedure described in the instructions for the questionnaire.

The survey items pertinent for this study included three sets of questions. First, the twins' genders, mutual age, and zygotic classification (identical or fraternal) were assessed. Almost all respondents reported that zygosity determination was based on the children's gender/appearance and afterbirth examination. These twinship assessments were accepted as valid because research has shown that judgments of zygosity based on how similar twins appear correspond almost perfectly with objective assessments based on blood tests. ${ }^{26} 27$

Second, the parent provided data on each child's asthma severity by estimating on five point rating scales the frequency $(0=$ 'never', $1=$ 'rarely', $2=$ 'occasionally', $3=$ 'fairly often', $4=$ 'very often') and intensity ( $0=$ 'never experience', $1=$ 'mild', 2='moderate', 3 ='fairly severe', $4=$ 'very severe') of asthma episodes the child would probably experience if he or she discontinued using relevant medication for a year (except for life threatening attacks). We

Descriptive statistics and correlation coefficients for trigger impact ratings among monozygotic and dizygotic twin pairs who are concordant for asthma

\begin{tabular}{|c|c|c|c|c|c|c|}
\hline \multirow[b]{2}{*}{ Triggers } & \multicolumn{3}{|l|}{ Monozygotic } & \multicolumn{3}{|l|}{ Dizygotic } \\
\hline & Mean (SD) & No & $\mathbf{r}$ & Mean (SD) & No & $\mathbf{r}$ \\
\hline Air pollution & $1.78(1.56)$ & 23 & 0.86 & $1.44(1.58)$ & 9 & $0 \cdot 77$ \\
\hline Allergy problems & $2.65(1.61)$ & 23 & 0.81 & $3.11(1.84)$ & 9 & $0 \cdot 79$ \\
\hline Cigarette smoke & $2 \cdot 13(1 \cdot 78)$ & 23 & 0.80 & $2.33(1.57)$ & 9 & $0 \cdot 37$ \\
\hline Stress or worry & $0.72(1.02)$ & 23 & 0.51 & $1.28(1.53)$ & 9 & $0 \cdot 88$ \\
\hline Anger & $0.61(0.89)$ & 23 & 0.76 & $1.44(1.73)$ & 9 & 0.55 \\
\hline Excitement & $0.91(1.12)$ & 23 & 0.56 & $1.56(1.77)$ & 9 & $0 \cdot 81$ \\
\hline Laughter & $0.74(1.07)$ & 23 & $0 \cdot 29$ & $1.39(1.61)$ & 9 & $0 \cdot 80$ \\
\hline High/low environmental temperature & $1.93(1.63)$ & 23 & 0.73 & $2 \cdot 10(1.71)$ & 8 & $0 \cdot 82$ \\
\hline High humidity & $1.96(1.98)$ & 23 & 0.97 & $2.04(1.83)$ & 8 & 0.95 \\
\hline Respiratory infection & $3.91(1.63)$ & 23 & 0.87 & $4.25(0.73)$ & 10 & $0 \cdot 29^{\star}$ \\
\hline Night time hours & $2.37(1.72)$ & 23 & 0.83 & $2.06(1.82)$ & 9 & 0.91 \\
\hline Physical activity & $2.61(1.90)$ & 23 & 0.84 & $3.17(2.07)$ & 9 & $0 \cdot 26^{\star}$ \\
\hline
\end{tabular}

${ }^{\star}$ Monozygotic and dizygotic correlations significantly different, $\mathrm{p}<0 \cdot 05$. asked the parents to make estimates of future episodes rather than past ones so that the parents would use recent knowledge to make ratings, thereby minimising accuracy problems that might have occurred if the estimates involved recollections to conditions that existed many years ago. These estimates were also designed to get assessments of the child's actual, real life asthma condition, without the severity being masked by effective medication. In classifying a child as asthmatic, we required that his or her survey data include: (1) ratings equal to or greater than 1 for both the frequency and intensity of asthma episodes and (2) an affirmative response to the question, 'At about what age was the child's asthma diagnosed by a physician?' Asthma severity was calculated as the product of the frequency and intensity ratings - an approach like that of the Psychosomatic Symptom Checklist ${ }^{28}$ for assessing illness severity.

The third set of pertinent questions applied only to children with asthma. These items assessed trigger impact by having the parents rate how often each of 12 'circumstances seem to accompany or contribute to' the child's asthma attacks. The circumstances were common asthma triggers ${ }^{21} 23$ - air pollution, allergy problems, cigarette smoke, stress or worry, anger, excitement, laughter, high or low environmental temperature, high humidity, respiratory infections, night time hours, and physical activity. The parents rated each trigger on a six point scale, with $0=$ 'virtually never' and 5='almost always'.

\section{Results}

Concordance rates for the presence of asthma among monozygotic and dizygotic twins were calculated by dividing the number of concordant pairs by the number of pairs in which one or both members were afflicted. In our data, 23 out of $39(58.97 \%)$ monozygotic pairs, compared with only 13 out of $55(23.64 \%)$ dizygotic pairs, were concordant for asthma. The ratio of these concordance rates is $2 \cdot 49: 1$. The mean (SD) age of asthma diagnosis by a physician was $2.4 \quad(2.8)$ years for the monozygotic twins and 3.6 (3.9) years for the dizygotic twins in our sample.

Analyses regarding genetic factors in asthma severity were restricted to data for concordant identical and fraternal twin pairs. The mean (SD) asthma severity ratings were $7 \cdot 13(4 \cdot 91)$ for the monozygotic twins and 5.37 (4.48) for the dizygotic twins, which suggests that most of the children had moderate asthma conditions. Using Pearson correlations and Fisher's $z_{\mathrm{r}}$ statistic ${ }^{29}$ to determine whether severity ratings were more strongly correlated for monozygotic than dizygotic twins, we found that asthma severity was significantly correlated for the monozygotic twins $(r=0.63$, $\mathrm{df}=21, \mathrm{p}<0.002)$ but not the dizygotic twins $(r=-0.12, \mathrm{df}=11, \mathrm{p}>0.70)$, and that the monozygotic twins' correlation coefficient for asthma severity was significantly different from that of the dizygotic twins $(p<0.03)$.

Analyses of genetic factors in the impact of 
specific triggers on asthma attacks also involved data only from concordant twin pairs. The table presents the descriptive statistics and correlation coefficients for each trigger separated by twin type. For both monozygotic and dizygotic twins, the highest mean impact ratings were for respiratory infection, physical activity, and allergy and the lowest were for the four emotion related triggers (stress or worry, anger, excitement, and laughter). Pearson correlational analyses revealed significant coefficients for the impacts of almost all triggers for both the monozygotic and dizygotic twins. However, the critical genetic analyses involved comparisons of monozygotic and dizygotic coefficients using Fisher's $z_{\mathrm{r}}$ statistic. These comparisons revealed significant differences in the correlations for monozygotic and dizygotic twins only for the triggers of respiratory infection ( $r \mathrm{~s}=0.87 v 0.29$, respectively, $\mathrm{p}<0.02)$ and physical activity $(r s=0.84 v 0 \cdot 26$, respectively, $\mathrm{p}<0.04$ ), suggesting that heredity may affect the action of respiratory infection and physical activity as triggers of asthma attacks.

\section{Discussion}

The results of the present study support and extend those of prior investigations regarding the role of genetic factors in asthma. Our finding that monozygotic twin pairs showed substantially higher concordance rates than dizygotic pairs for the presence of asthma confirms the role of heredity found in earlier studies. Although the concordance rates for monozygotic and dizygotic twins were relatively high in the present sample, our ratio of monozygotic to dizygotic concordance percentages was $2 \cdot 49: 1$, which is comparable with the ratios found in the earlier studies: $3 \cdot 96: 1,6$ $2 \cdot 41: 1,{ }^{7}$ and $1 \cdot 86: 1 .^{8}$ These ratios suggest a clear but moderate role of heredity in asthma aetiology. Environmental factors also affect the development of asthma, as evidenced by research findings that viral infections during infancy and long term exposure to airborne irritants increase future asthma symptoms. ${ }^{121}$

\section{ASTHMA SEVERITY AND TRIGGERS}

The current findings extend our knowledge about the role of genetic factors in asthma in two ways. First, the finding that asthma severity is more similar among monozygotic than dizygotic twins is new to the asthma literature, as is our method for assessing the severity of this illness: using the product of rated frequency and intensity of illness episodes. Our results indicate that the role of heredity in asthma may include specific aspects that influence how serious the condition becomes. This possibility is consistent with the recent findings ${ }^{20}$ that an aspect of genetic information, that is, the size of a causal fragment, affects the severity of a hereditary disease.

Second, our study found evidence that heredity influences the role of respiratory infection and physical activity as triggers of asthma attacks. The link between heredity and respiratory infection as an asthma trigger may help to explain why most infants and toddlers who develop episodes of wheezing when they have viral respiratory infections do not develop asthma later in childhood. ${ }^{30}$ Although wheezing is a major diagnostic symptom of asthma, ${ }^{31}$ it may be that infection related wheeze is different from asthma and that individuals who develop both infection related wheeze and asthma have certain genetically determined features that link the phenomena. Our finding that heredity plays a part in physical activity as an asthma trigger may help to explain why asthmatic individuals differ in the strength of reaction to exercise tests.

The links we found between heredity and the impact of two asthma triggers raise important questions. For example, what processes underlie the link between genetics and these triggers? Although research has not yet determined the mechanisms by which respiratory infection and physical activity lead to an asthma attack, several likely routes have been outlined. ${ }^{24}$ Two of these routes apply to both respiratory infection and physical activity as triggers. One route involves heightened release of bronchospastic and inflammatory mediators in the airways, and the other route involves changes or damage to the airway epithelium. Perhaps the genetic factors that underlie the triggering impact of respiratory infection and physical activity affect mediator release and/or epithelial characteristics.

Another question our findings raise is why our data revealed no genetic effects for the 10 other triggers. One reason for this outcome may be that the genetic effects in asthma are specific mainly to the triggering processes that accompany respiratory infection and physical activity. Secondly, the small number of dizygotic twins in the trigger analyses made it difficult to find statistically significant differences between monozygotic and dizygotic correlations. A third reason may have to do with the differences in trigger impact reflected in our data. Respiratory infection had the highest rated impact (overall $M=4 \cdot 02$ ); physical activity and allergy problems were tied for second place (overall $M s=2 \cdot 77$ and $2 \cdot 78$, respectively), followed by night time hours. Environmental conditions and emotion related circumstances had much lower trigger impact ratings. These differences in ratings may reflect real differences in trigger impacts, or they may reflect cognitive biases of the respondents, which might result from parents' awareness of a trigger's presence during their children's attacks or the parents' expectations about each trigger's impact. But our data do not show any clear signs of cognitive biases - for instance, the SDs in the table are fairly similar for triggers that are easily apparent, such as the child's symptoms of infections or allergy problems, and triggers that are more obscure, such as the child's stress or worry.

\section{INTERPRETATIVE ISSUES}

The findings that genetic factors may influence asthma severity and the impact of some 
triggers of attacks are provocative but must be interpreted cautiously for at least two reasons. First, the measures of asthma severity and trigger impact are new to the literature on asthma. Second, the methodology we used was relatively exploratory in nature, allowing data collection on a large sample of subjects for many variables at low cost but producing some limitations in the interpretation of our findings.

One interpretative limitation is that data collected through questionnaires are always subject to concerns about their validity and reliability. Inaccuracies can result from respondents' biased reporting and their incorrect knowledge and retrospective memories, particularly when providing information on individuals other than themselves. But survey methods in asthma research have some important strengths, can be quite accurate, and may be particularly appropriate and useful when studying atopic illnesses because symptoms occur episodically. ${ }^{32-34}$

In an effort to minimise problems in the reliability and validity of our data, we made the questions as easy and objective to answer as possible. We also instructed the respondents to 'fill out the questions and pages in their presented order and answer each question as carefully and objectively as you can' and 'when answering questions about each individual, do not compare your answers to those you gave for other family members'. Questions regarding each child were on separate pages. Moreover, the respondents were members of an organisation that views research participation as a central mission. How effective these factors were in reducing data inaccuracies is unclear. But the pattern of significant and nonsignificant effects we found suggests that our methodology may have minimised potential responder biases, such as respondents' tendencies to apply cognitive 'sets' that could consistently either exaggerate or reduce their ratings of illness characteristics within or between individuals. For example, our trigger impact results (see table) revealed significantly higher correlations for monozygotic than dizygotic twins for two triggers, but not the remaining ones - and for a few triggers, the dizygotic twins had markedly, but not significantly, higher correlations.

Another interpretative limitation stems from relying on data collection from one respondent (virtually always the mother), without corroboration from other sources. Although having an independent source to corroborate the mothers' reports might have strengthened the data set, no practical or reliable source was available. To enhance the validity and reliability of the parents' data, we required that they affirm a physician's diagnosis of asthma and asked those respondents who filled out the survey at home to consult their children aged 10 or older in answering the questions about the children's asthma. It may also be that having a single respondent report on both members of each twin pair promoted internal consistency in the way the parents interpreted the questions and used the rating scales.
In conclusion, we used survey methodology to compare monozygotic and dizygotic twins with regard to the presence and severity of their asthma and the impact of 12 common triggers of attacks. Our results suggest that heredity affects whether or not an individual will develop asthma, the severity of the person's asthma condition, and the impact of respiratory infection and physical activity as triggers of asthma attacks.

We gratefully acknowledge the help of the National We gratefully acknowledge the help of the National Organization of Mothers of Twins Clubs in distributing the
survey; Nancy Breland and Margaret Ruddy for suggestions on survey; Nancy Breland and Margaret Ruddy for suggestions on designing the questionnaire; and Christian McCarth
Georg Ugi for work in creating the computer data base.

Georg Ugi for work in creating the computer data base.
This research was supported in part by a grant from the This research was supported in part by a grant from the
Faculty and Institutional Research and Sabbatical Leave Committee of Trenton State College. Part of these data were presented at the March 1993 meetings of the Society of Behavioral Medicine in San Francisco. Jarrett Goldfedder is currently a doctoral student at Ohio University.

1 Busse WW. Respiratory infections: their role in airway responsiveness and the pathogenesis of asthma. 7 Allergy Clin Immunol 1990; 85: 671-83.

2 Asthma and Allergy Foundation of America. Allergy and allergic diseases: recent statistics. Washington: The Foundation, 1992 .

3 Cluss PA, Fireman P. Recent trends in asthma research Annals of Behavioral Medicine 1985; 7 (4): 11-6.

4 Gergen PJ, Mullally DI, Evans R. National survey of prevalence of asthma among children in the United States, 1976 to 1980 . Pediatrics $1988 ; 81$ : 1-7.

5 Duffy DL, Martin NG, Battistutta D, Hopper JL, Mathews JD. Genetics of asthma and hay fever in Australian twins. Am Rev Respir Dis 1990; 142: 1351-8.

6 Edfors-Lubs ML. Allergy in 7000 twin pairs. Acta Allergologica 1971; 26: 249-85.

7 Hopper JL, Hannah MC, Macaskill GT, Mathews JD. Twin concordance for a binary trait: III. A bivariate analysis of hay fever and asthma. Genet Epidemiol 1990; 7 277-89.

8 Nieminen MM, Kaprio J, Koskenvuo M. A populationbased study of bronchial asthma in adult twin pairs. Chest 1991; 100: 70-5.

9 Higgins $M$, Keller J. Familial occurrence of chronic respiratory disease and familial resemblance in ventilatory

10 Leeder SR, Corkhill RT, Wysocki MJ, Holland WW, Colley JRT. Influence of personal and family factors on ventilatory function of children. British fournal of Preventive and Social Medicine 1976; 30: 219-24.

11 Bazaral M, Orgel HA, Hamburger RN. Genetics of IgE and allergy: serum IgE levels in twins. $\mathcal{F}$ Allergy Clin Immuno 1974; 54: 288-304

2 Burrows B, Martinez FD, Halonen M, Barbee RA, Cline MG. Association of asthma with serum IgE levels and skin-test reactivity to allergens. $N$ Engl $¥$ Med $1989 ; 320$ 271-7.

13 Sistonen P, Johnsson V, Koskenvuo M, Aho K. Serum IgE levels in twins. Hum Hered 1980; 30: 155-8.

14 Townley RG, Southard JG, Radford P, Hopp RJ, Bewtra AK, Ford L. Association of MS Pi phenotype with airway hyperresponsiveness. Chest 1990; 98: 594-9.

15 König P, Godfrey S. Prevalence of exercise-induced bronchial lability in families of children with asthma. Arch Dis Child 1973; 48: 513-8.

16 König P, Godfrey S. Exercise-induced bronchial lability in monozygotic (identical) and dizygotic (nonidentical) monozygotic (identical) and dizygotic (no

17 Sinha SB, Yadav CN. The asthmogenic effect of exercise stress on the normal first degree relatives of asthmatic subjects. Indian $\mathcal{F}$ Chest Dis Allied Sci 1985; 27: 141-7.

18 Hopp RJ, Bewtra AK, Watt GD, Nair NM, Townley RG. Genetic analysis of allergic disease in twins. $\mathcal{f}$ Allergy Clin Immunol 1988; 73: 265-70.

19 Longo G, Strinati R, Poli F, Fumi F. Genetic factors in nonspecific bronchial hyperreactivity. Am $\mathcal{f}$ Dis Child 1987; 141: 331-4.

20 Harley HG, Brook JD, Rundle SA, et al. Expansion of an unstable DNA region and phenotypic variation in myotonic dystrophy. Nature 1992; 355: 545-6.

21 Cropp GJA. Special features of asthma in children. Chest 1985; 87 (suppl 1): 55-62S.

22 Evans $R$. What you should know about children's asthma. Asthma and Allergy Advance 1990; Aug/Sept (reprint): 1-4.

23 Janson-Bjerklie S, Carrieri VK, Hudes M. The sensations of pulmonary dyspnea. Nurs Res 1986 ; 35: 154-9.

24 pulmonary dyspnea. Nurs Res 1986; 35: 154-9. 48: 169-78.

25 Barbee RA, Dodge R, Lebowitz ML, Burrows B. The epidemiology of asthma. Chest 1985;87 (suppl 1): $21-5 \mathrm{~S}$

26 Kasriel J, Eaves L. The zygosity of twins: further evidence on the agreement between diagnosis by blood groups and on the agreement between diagnosis by blood groups
written questionnaires. F Biosoc Sci 1976; 8: 263-6.

27 Sarna S, Kaprio J, Sistonen P, Koskenvuo M. Diagnosis of twin zygosity by mailed questionnaire. Hum Hered 1978, 28: 241-54. 
28 Chibnall JT, Tait RC. The psychosomatic symptom checklist revisited: reliability and validity in a chronic pain population. $\mathcal{F}$ Behav Med 1989; 12: 297-307.

29 Ferouson G. Statistical analysis in psychology and education. 5 th Ed. New York: McGraw-Hill, 1981.

30 Martinez FD, Wright AL, Taussig LM, et al. Asthma and wheezing in the first six years of life. $N$ Engl f Med 1995; 332: 133-8.

31 Weinberger $M$. Managing asthma. Baltimore: Williams and Wilkins, 1990.
32 Dold S, Wjst M, von Mutius E, Reitmeir P, Stiepel E. Genetic risk for asthma, allergic dermatitis. Arch Dis Child 1992; 67: 1018-22.

33 Burney P, Chinn S. Developing a new questionnaire for measuring the prevalence and distribution of asthma. Chest 1987; 91 (suppl 6): 79-83S.

34 Backer V, Ulrik CS, Hansen KK, Laursen EM, Dirksen A, Bach-Mortensen N. Atopy and bronchial responsiveness in a random population sample of 527 children and adolescents. Ann Allergy 1992; 69: 116-22. 